International Journal of Engineering \& Technology, 7 (4.10) (2018) $908-912$
International Journal of Engineering \& Technology
SPC
Website: www.sciencepubco.com/index.php/IJET
Research paper

\title{
Network Border Patrol: Prevent Profusion Crash and Prating Decency in the Network
}

\author{
B. Pawan Kumar Kurmi ${ }^{1}$, Rahul Jain ${ }^{1}$, K. Dheeraj Reddy ${ }^{3}$, GSGN Anjaneyulu ${ }^{4}{ }^{4}$ \\ ${ }^{1}$ Project Engineer, WIPRO Technology Ltd, Raheja Mindspace, Airoli Navi Mumbai \\ ${ }^{2}$ Associate Cloud Engineer, ATOS GLOBAL IT Solution And Services Pvt Ltd, Vadodara, Gujarat \\ ${ }^{3}$ B.Tech, School of Computing Science and Engineering, VIT, Vellore, Tamilnadu, India \\ ${ }^{4}$ Professor, Dept. of Mathematics, SAS, VIT, Vellore, Tamilnadu \\ Corresponding Author E-mail: pawankumarkurmi@gmail.com
}

\begin{abstract}
The process to process pattern of online network mobbing flow control is a significant constituent in its scalability metrics and heftiness altercation no protocol, mechanism, or ability must be given into the Internet. In this article, we propose and investigate new approach congestion avoidance approach is known as Network Border Patrol (NBP).Process to process congestion traffic control is only single approach; NBP trusts on the conversion and evaluation in the middle of routers at the rims of a network. This depends on priority to identify and restrict not responding traffic flux before they enter the network. However, they are incompetent to avert the jamming disintegration, but damage produced by claims that are insensitive to network online blocking to report the above malady problem.
\end{abstract}

Keywords: Border Regulator, Border Observer, Congestion Switch, Congestion Disintegration, CoreStateless Devices, Process To Process Dispute, Internet.

\section{Introduction}

If we implement the existing approach, then we find some bugs on the network, because this approach is not sufficient to handle from many to many data transferable nodes as data could not be reliable and more time consuming. So we inebriated this recent approach and we debug that bugs. So we have a new system that provides the solutions of existing bugs. In this technique, data should be reliable and more secure, traffic prevent, efficient congestion avoidance mechanisms compared to earlier approach. The current approach is much better and will take less time for execution.

This section elaborates three important facts of the NBP approach:

1. The architectural components, namely the tailored periphery routers, which must be there in the set of connections.

2. The input control calculation, which decides how and when data is traded between edge switches.

3. The rate of manipulation and calculation, that utilizes the data communicated as a portion of criticism packages to manage stream transmission rates and in this method circumvent blockage crumbling in the system.

\subsection{Related work}

The illnesses of blockage breakdown from undelivered packages and of unreasonable data transfer capacity allocations have not gone unrecognized. A little have contended that there are communal impetuses for sight and sound requests to be cordial to the arrangement, as a request should not have each desire to be believed as in price of throughput debasement in the Internet. Of sequence, malignant disavowal of-administration aggressions employing lethargic UDP flows are becoming to be stunning so- journ in the Internet and they are an illustration that the Internet cannot depend exceptionally on communal impetuses to manipulation blockage or to work decently. A little have contended that these illnesses could be arbitrated across the use of enhanced package arranging or line administration.

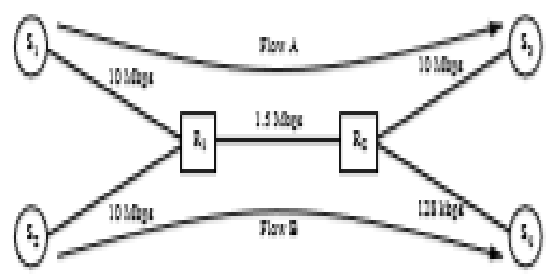

Fig. 1: Network which experience congestion collapse

Arrangements in framework switch. For instance, every flux packet arranging devices like Weighted Fair Queuing (WFQ), challenge to deal impartial distributions of band width to flux struggling for the matching bond. Several approaches came to avoid the congestion collapse. Their endorsed methodology involves chose access switch to examine huge bandwidth streams so as to regulate whether those are reactive to jamming. However, they cannot identify the stream rates and unreactive streams. They are fairly random and are not permanently fruitful. Design of the ATM existing bit rate amenity and involve all system switches to determine fair allocation of the existing internet, since they Pilate the 
network composition way of thinking. Then to switch implementations as simple and pushing complexity to the edges.

\subsection{Demerits with Current Structure}

1. Data Sachets are cushioned in the switches show in the system which grounds clogging, crumbling from detained bundles emerges when transmission capacity is constantly devoured by parcels that are throw down before coming to their definitive destination.

2. Retransmission of detained Sachets is necessary to guarantee no damage of information. Imbalanced bandwidth sharing rises in the internet because of the vicinity of detained packets.

\subsection{Motivation}

In the old model there were only two sources, which led to data collision and loss of information. Hence such results are more time consumption and data loss. So we now intrigued to implement a new model, which could overcome those drawbacks. Actually there are just two sources which prompt the information impact and the loss of data. Thus the results require finally additional time and information misfortune. So we would like to introduce another module in the system such that the system performance could be upgraded by without loss of data, with less time consumption and accuracy in transforming data.

\section{Proposed work}

\subsection{System Design:-}

Module 1 Module 2 Module 3 Module 4 Module 5

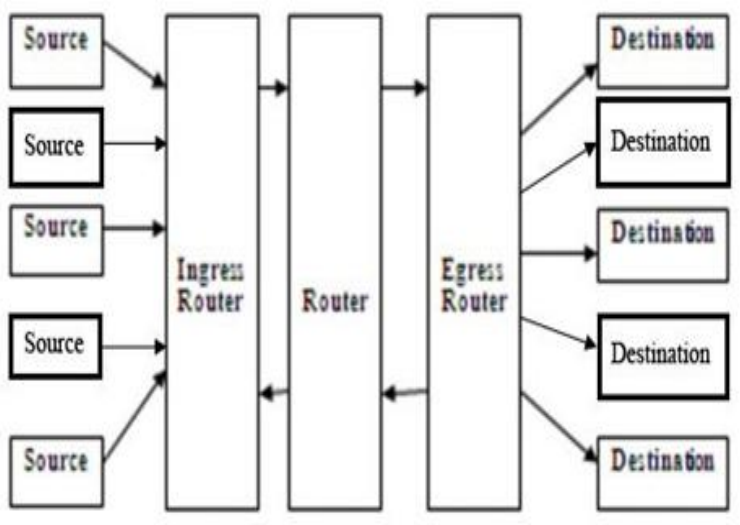

Fig. 2: Flow of DATA in the NETWORK

\subsection{System Architectural Features}

As a consequence of its compulsion obedience to end-to-end clogging mechanism, the existing Internet encounters a couple of illnesses: Cramming breakdown from detained parcels and out of line designations of data transfer capacity between contending movement streams.

The principal disease blockage breakdown from undelivered parcels emerges when bundles that are dropped before coming to their definitive constantly devour transmission capacity destinations. The second ailment -unreasonable data transmission allotment to contending system streams emerges in the Internet for an assortment of details, one of which is in the existence of utilizations that don't react legitimately to blockage. Versatile applications (e.g.. TCP-based applications) that react to clogging by quickly dropping their broadcasting rate are accusable to take delivery of unethically slight bandwidth sharing when competing with insensitive applications. The Internet based protocols themselves can initiate grievances.

\subsubsection{An NBP Ingress Router}

The structural planning of a NBP departure switch's data port. Bundles sent by entrance switches land at the data port of the departure switch and are initially ordered by stream. On account of IPv6, this is finished by analyzing the bundle header's stream mark, though on account of IPv4, it is finished by inspecting the parcel's origin and terminus addresses and port numbers. Each stream's bit rate is utilizing a rate estimation calculation, for example, the Time Sliding Window (TSW).

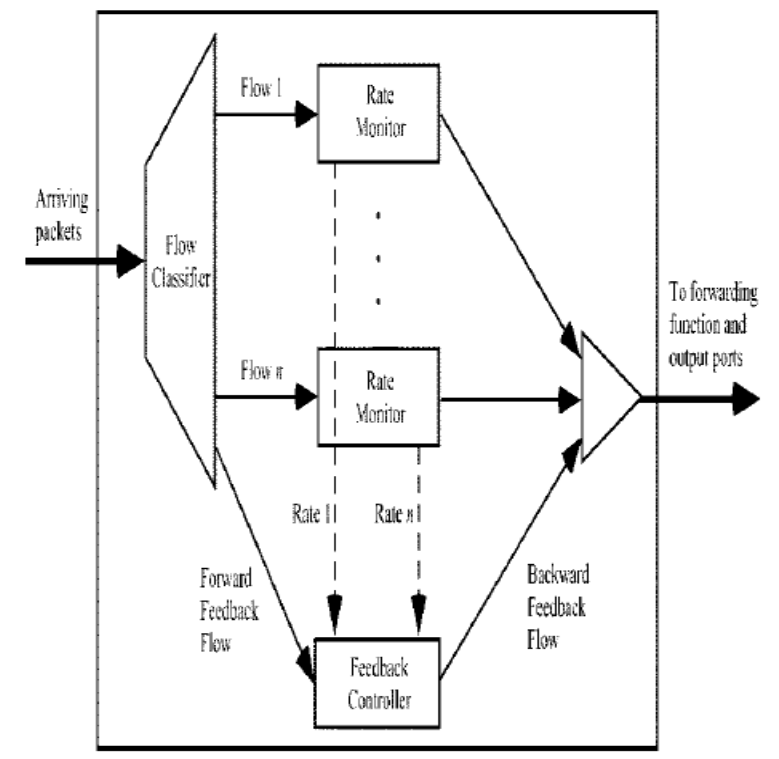

Fig. 3: NBP Ingress Router

These ratings are gathered by a criticism controller, which returns them in reverse input bundles to an entrance switch at whatever point a forward input parcel touches base from that entrance switch. Now and again, to be portrayed later in this segment, in reverse input parcels are likewise produced no concurrently; that is, a departure switch sends them to an entrance switch without first sitting tight for a forward criticism bundle.

\subsubsection{An NBP Egress Router}

The yield ports of NBP entrance switches are additionally upgraded. They contain a stream classifier, every stream movement shapers (e.g., defective cans), a criticism controller, and a rate controller. This can be seen in Figure 4. The stream analyzer gatherings packages into streams and the activity figures restrain the tariffs at which bundles from each individual streams enter the system.

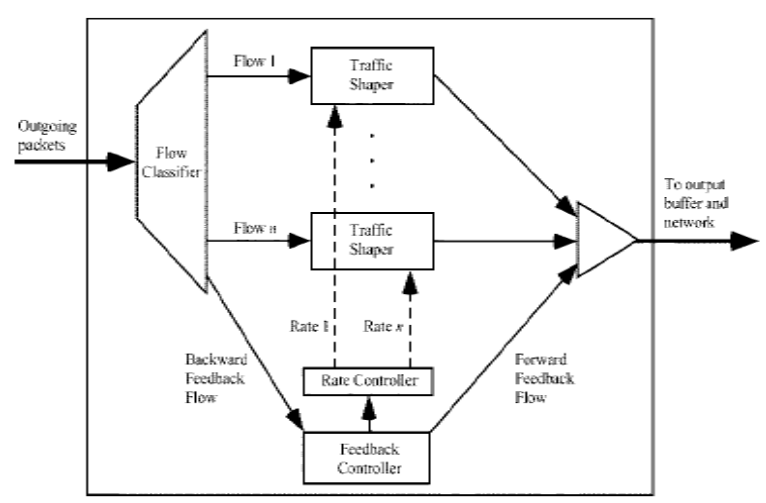

Fig. 4: NBP Egress Router

The input controller gets in reverse criticism bundles coming back from departure switches and permits their substance to the rate regulator. It likewise creates forward input bundles, which it 
sometimes communicates to the system's departure switches. The rate regulator confirms movement figures parameters as per a TCP-like rate regulate calculation, which is portrayed later in this area.

\subsection{Benefits of Projected System}

To keep the Crowding Control through the correspondence over system we are presenting Network Border Patrol Theory. The Benefits over these concepts are recorded underneath:

Cushioning of bundles in completed in the edge switches as opposed to in the center switches. The bundles are sent into the system in view of the limit of the system and thus there is no probability of any undelivered parcels show in the system. Nonattendance of undelivered packages evades over-weight because of retransmission. Reasonable designation of data transmission is guaranteed.

\section{Execution}

\subsection{Caliber Vignette}

The several Calibers in this structure are given below-

Module 1:- Source Component.

Module 2:- Ingress Router Component.

Module 3:- Router Component.

Module 4:- Egress Router Component.

Module 5:- Destination Component

\subsubsection{Source Component}

The undertaking of this Component is to transmit the bundle to the Ingress switch.

Information Substances: Message to be communicated from the source to the terminus hub as bundle with IP address for its recognizable proof..

Algorithm: Triple DES (Data Encryption Standard).

Output: Configured bundle with the obliged data for imparting between the source $\&$ the terminus hub.

\subsubsection{Ingress Router Component}

A power switch working on a stream going into a system is called an entrance switch. Control Rate permits an entrance switch to police the rate at which each stream's bundles enter the system. Utilizing control rate and whole basic calculation to rank the hubs in the system.

Information Substances: Which focus the rate of the packets. Algorithm: Per-stream Traffic Shapers (e.g., Leaky Bucket Algorithm), A Controller Feedback, Controller Rate.

Output: All the hubs in the system or network allotted with a remarkable rank.

\subsubsection{Router Component}

The errand of this Component is to acknowledge the parcel from the Ingress switch and send it to the Egress switch.

\section{Information Substances}

Gets information from neighboring hubs and move into other neighboring hubs

Output: Exchange bundles to neighboring hubs

\subsubsection{Egress Router Component}

An edge switch working on a stream going out of a system is called a departure switch. Rate observing permits a departure switch to decide how quickly each streams bundles are parting from the system. Utilizing time sliding window and rate checking calculation to rank the hubs in the system. Information substances-which focus the rate of the data packets stream in the system. Algorithm: Time Sliding Window (TSW) Algorithm, a Controller Feedback, and Monitor Rate.

Output: packets are delivering to the destination.

\subsubsection{Destination Component}

The assignment of this Module is to acknowledge the parcel from the Egress switch and put away in a document in the Destination machine.

Information substances: message to be collected from the Egress router to the terminus node in the form of data packets with IP address.

\section{Security Analysis}

We actualized that systems then we discovered the new security access. Security is most imperative thing on the current web arrange on virtual world. With the cutting edge pattern, security is imperious since the vast majority of the world is utilizing web system to information. Different development exploration has been done for the encryption of information which was not discovered so encouraging by giving the system security. In this paper, we had proposed a thought of encryption and unscrambling calculation which give successful and sufficient web system security.

In these strategies framework or information secure is more contrast with existing framework. We can evacuate the activity, information exchange rate and enhance security. On the off chance that takes after that approach, we could get the yield and its lessened time multifaceted in nature.

\section{Implementation and Results}

We are transmitting the information through entering the content or skimming the content record by giving encryption methodology for security reason. In the inflowing of encryption key must not be as much as are equivalent to each other.

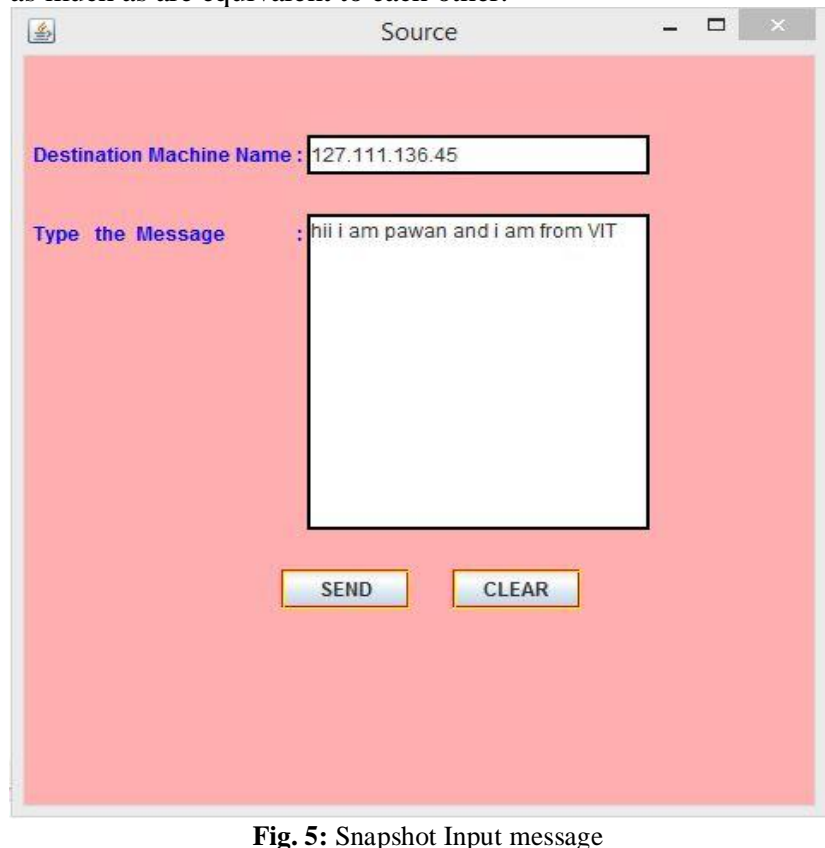

It accepts the information from the source as parcels. Before sending the bundle to the switch it will trade the input in the middle of entrance and departure routers. In the wake of getting in reverse input from the departure switch it will send the bundles to the center switch. It will simply gets the bundles from the entrance 
switch and send those parcels straightforwardly to the departure switch.

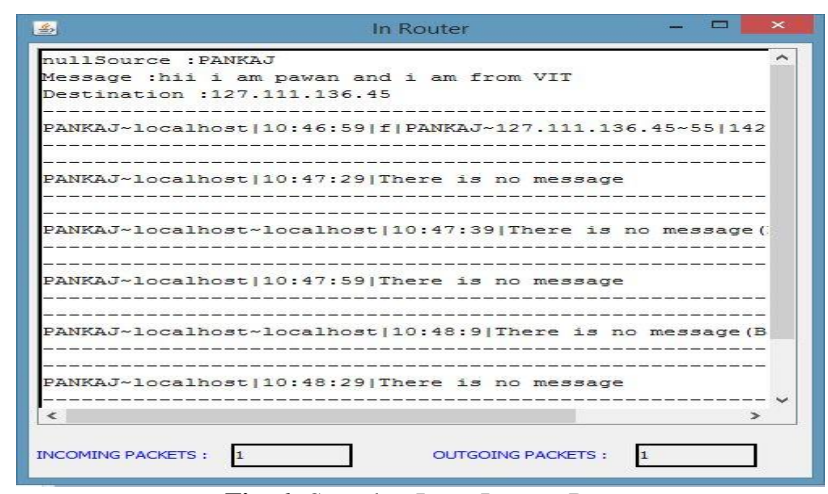

Fig. 6: Snapshot Input Ingress Router

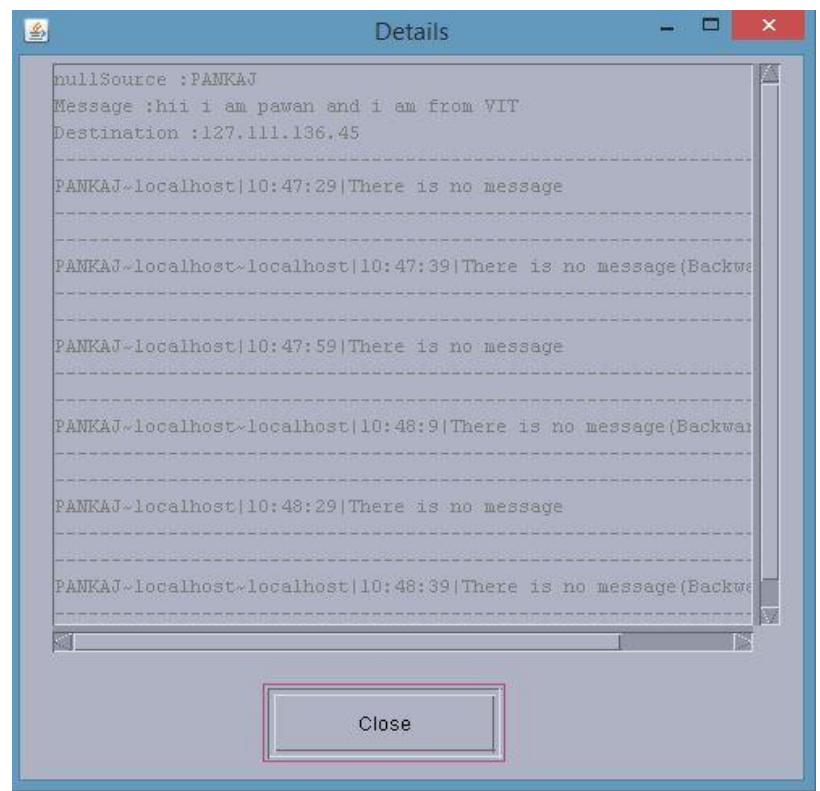

Fig. 7: Snapshot Router Monitoring

The departures switch observing the rate of the bundles in a system. In view of the rate of every stream it will send to the entrance switch as a regressive input furthermore getting bundles from the center switch and send those parcels sand to the destination.

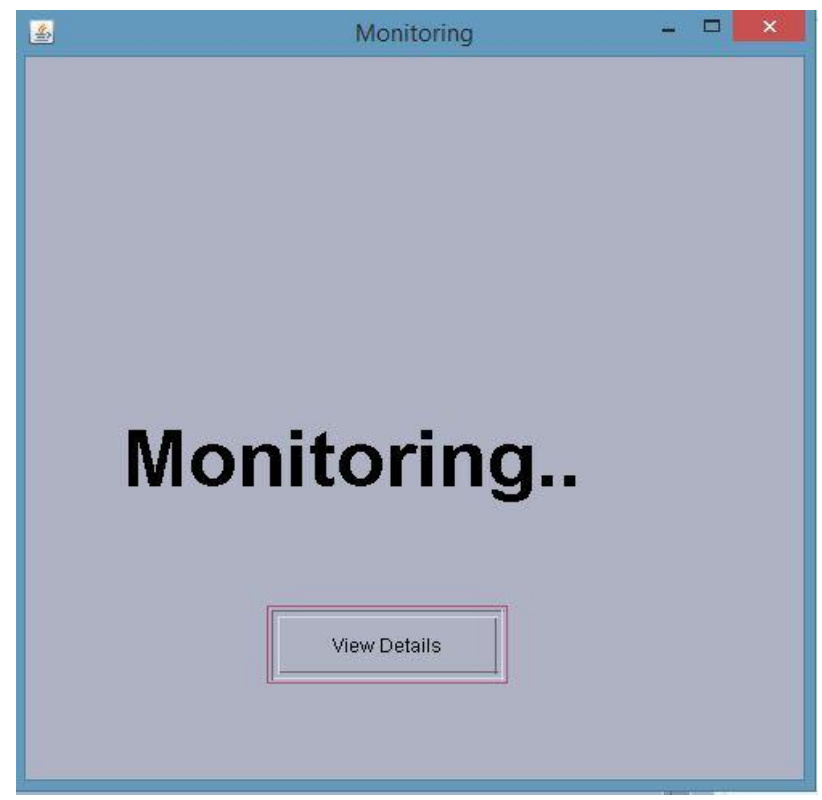

Fig. 8: Snapshot Router Monitoring

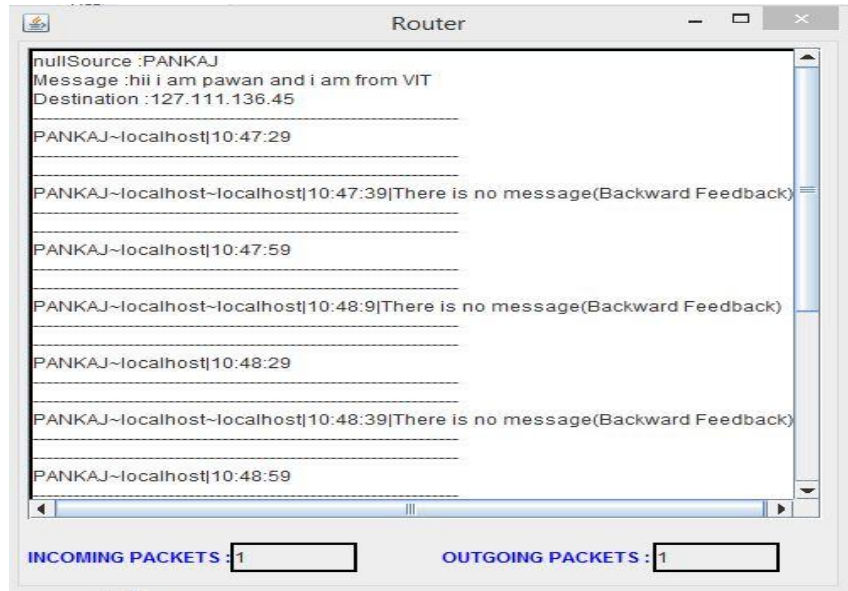

Fig. 9: Snapshot Egress Router

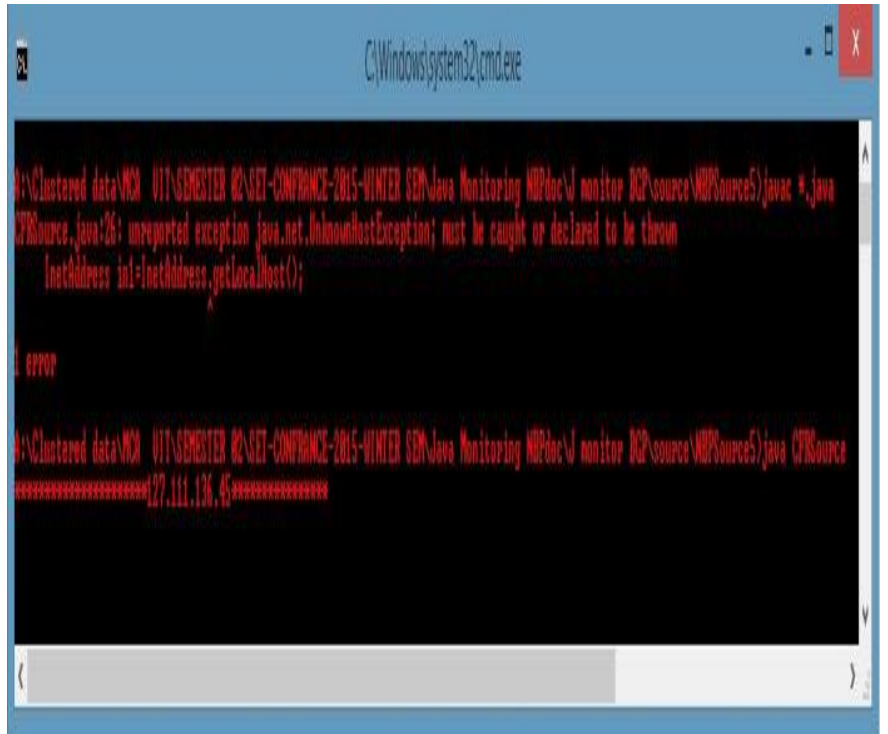

Fig. 10: Execution part of Ingress Router

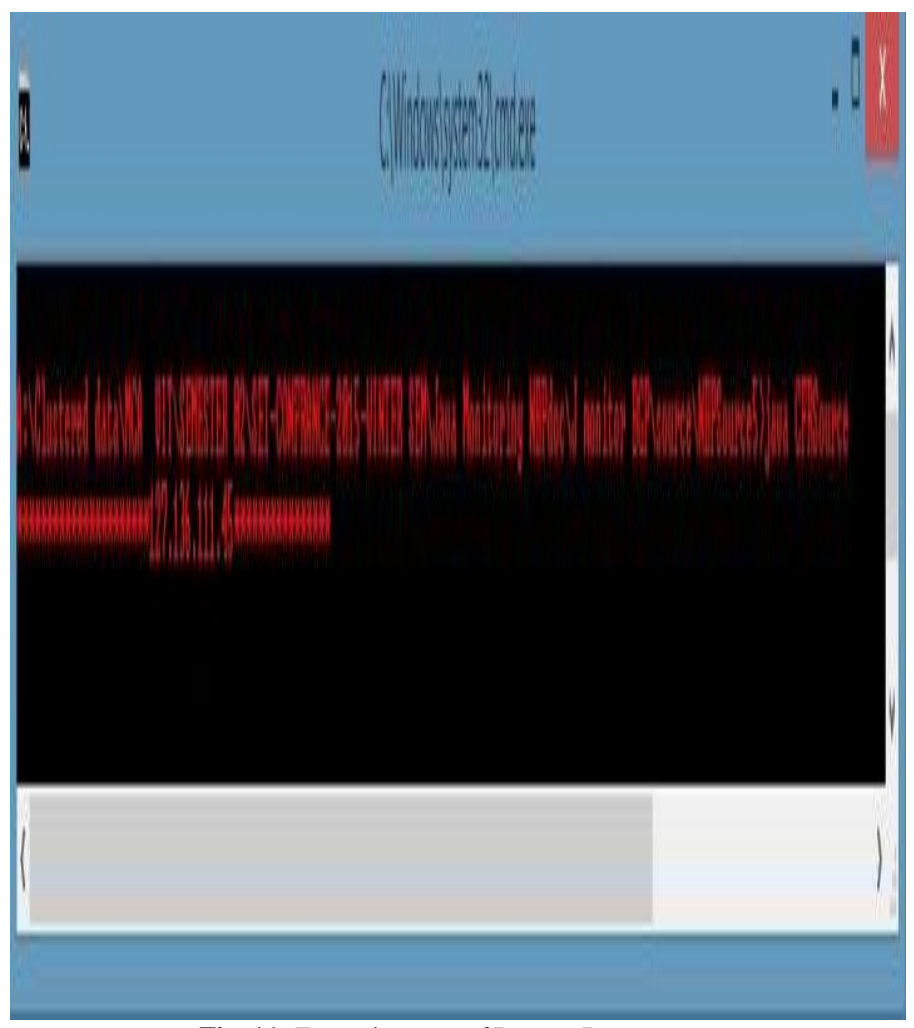

Fig. 11: Execution part of Ingress Router 


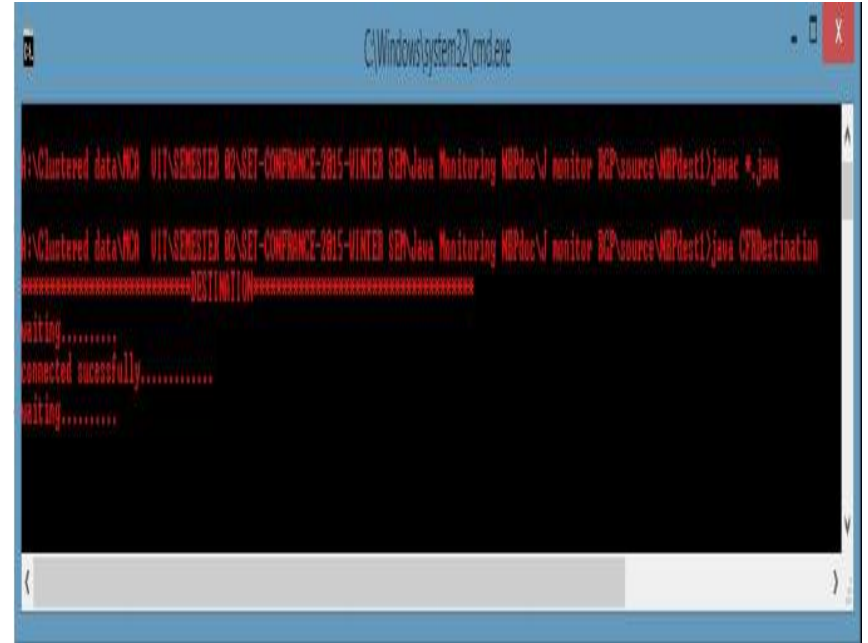

Fig 12: Execution part of Egress router

\section{.}

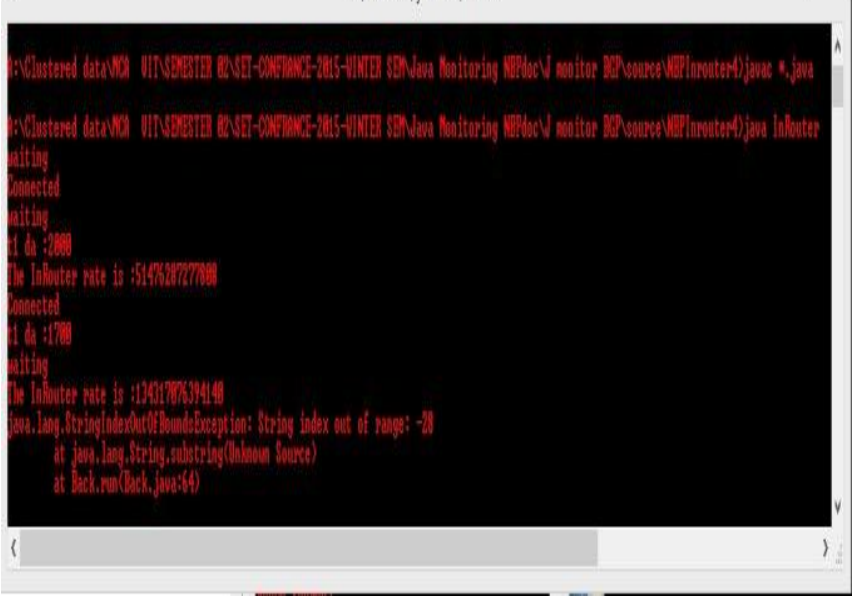

Fig. 13: Execution Part of Router

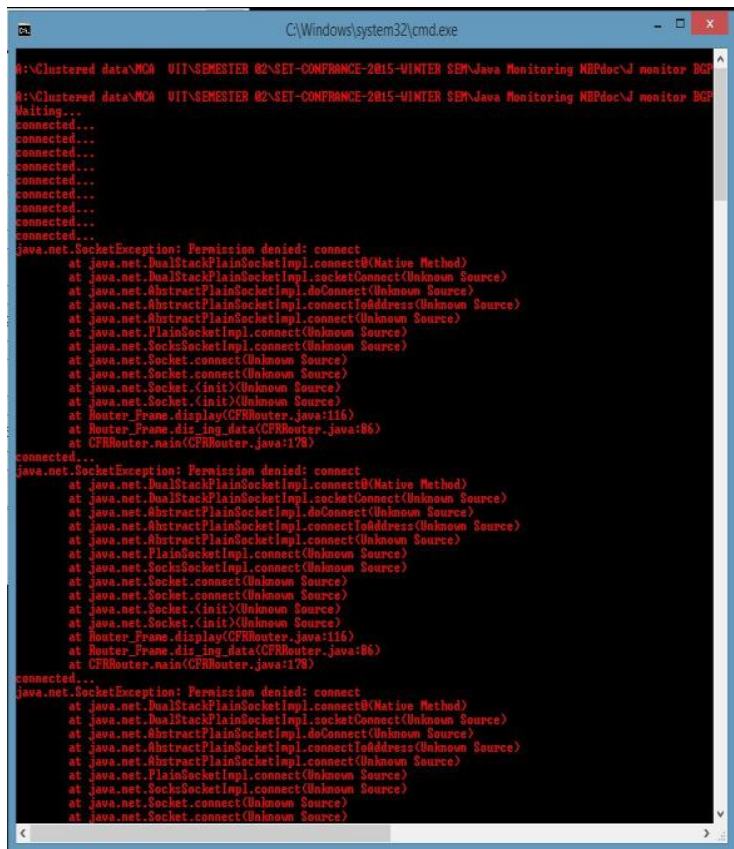

Fig. 14: Execution part of Router Monitoring

We are getting the bundles from the Egress switch and decoded the message utilizing the unscrambling Process and after that the information send by the source will be demonstrated in the content zone determined in the destination.

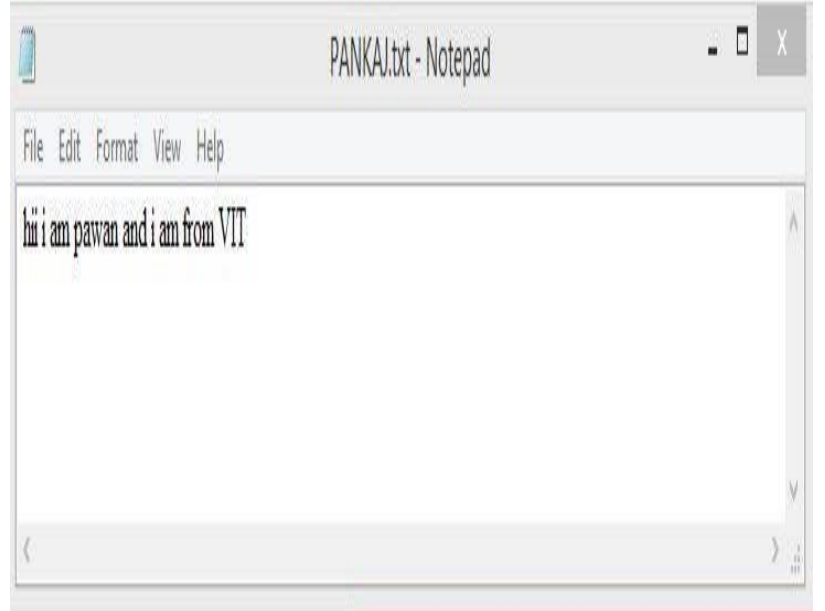

Fig. 15: Snapshot Notepad Message- Receiver Side

\section{Conclusion}

In this system, we have displayed a novel blockage shirking instrument for the Internet called Network Border Patrol and an upgraded center stateless reasonable lining component. Not at all like standing Internet clogging control approaches, which depend exclusively on end-to-end control, has NBP had the capacity to keep blockage breakdown from non-transmitted parcels. It does this by guaranteeing at the outskirt of the system that each flow parcel doesn't enter the system quicker than they find themselves able to abandon it. NBP obliges no changes to center switches or to end frameworks.

Only edge switches are upgraded with the goal that they can accomplish the imperative every flow checking, every flow rate control and input trade tasks. Broad recreation results gave in this paper demonstrates that NBP effectively keeps blockage breakdown from undelivered bundles. They likewise demonstrate that, while NBP is not able to dispense with injustice all alone, it has the capacity accomplish surmised worldwide max-min decency for contending system flows. They uneven world wide max-min decency in a totally centered state.

\section{References}

[1] Van Jacobson, "Congestion avoidance and control," ACM Computer Communications Review, vol. 18, no. 4, pp. 314-329, Aug. 1988, Proceedings of the Sigcomm '88 Symposium in Stanford, CA, August, 1988.

[2] Demers.A, Keshav.S, and Shenker.S, "Analysis and Simulation of a Fair Queueing Algorithm," in Proc. of ACM SIGCOMM, September 1989, pp. 1-12.

[3] Parekh.A, Gallager.R "A General Approach to Flow Control - the Single Node Case," IEEE/ACM Transactions on Networking, vol. 1, no. 3, pp. 344-357, June 1993.

[4] Stoica, Shenker.S, and Zhang.H, "Center Stateless Fair Queueing Achieving Approximately Fair Bandwidth Allocations in High Speed Networks," in Proc. of ACM SIGCOMM, September 1998, pp. 118-130.

[5] Cao.Z, Wang.Z, and Zegura.E, "Rainbow Fair Queuing: Fair Bandwidth Sharing Without Per-Flow State," in Proc. of IEEE Infocom '2000, March 2000.

[6] Container.R, Prabhakar.B, and Psounis.K, "Gag - A stateless dynamic line administration plan for approximating reasonable transfer speed assignment," in Proc. of IEEE Infocom '2000, March 2000.

[7] Suter.B, Lakshman.T.V, Stiliadis.D and Choudhury.A, "Outline Considerations for Supporting TCP with Per-Flow Queueing," in Proc. of IEEE Infocom '98, March 1998, pp. 299-305.

[8] Braden.B, Clark.D, "Recommendations on Queue Management and Congestion Avoidance in the Internet," RFC 2309, IETF, April 1998, https://tools.ietf.org/pdf/rfc2309.pdf. 